\title{
An investigation of experimental myasthenia gravis
}

\author{
S. F. JONES, J. L. BRENNAN, AND J. G. MCLEOD \\ From the Department of Medicine, University of Sydney, N.S.W., Australia
}

SUMMARY Guinea-pigs were immunized with antigen prepared from calf thymus and muscle, and from guinea-pig thymus, and rats were immunized with antigen prepared from rat thymus and rat muscle. There was an increased incidence of delayed hypersensitivity and circulating thymus antibodies in the immunized guinea-pigs and an increased incidence of thymitis in the immunized guinea-pigs and rats. However, when compared with control animals, there was no electrophysiological evidence of impairment of neuromuscular transmission in the immunized animals.

In 1966, Goldstein and Whittingham reported that guinea-pigs immunized with an emulsion of calf thymus or muscle in Freund's complete adjuvant (FCA) developed a defect in neuromuscular transmission of the type found in myasthenia gravis. In these guinea-pigs there were changes in thymic histology, interpreted by Goldstein and Whittingham (1966) as autoimmune thymitis. They suggested that autoimmune thymitis in guinea-pigs represented an experimental model of myasthenia gravis, and subsequent experiments on guinea-pigs and rats by Goldstein and his co-workers (Goldstein and Hofmann, 1968; Goldstein, Strauss, and Pickeral, 1969) and by Kalden, Williamson, Johnston, and Irvine (1969) have supported this hypothesis. By contrast, Vetters, Simpson, and Folkarde (1969), and Kaufman, Rushworth, and Wright (1969) were unable to detect any abnormality in neuromuscular transmission in guinea-pigs immunized in the manner described by Goldstein and Whittingham (1966).

The present paper describes the results of investigations in which attempts were made to produce an experimental model of myasthenia gravis in guinea-pigs and rats by immunological methods.

\section{METHODS}

ANIMALS Outbred guinea-pigs weighing 300-550 g, outbred Wistar rats weighing 150-220 g, and inbred Lewis rats of weight approximately $140 \mathrm{~g}$, were used in the various experiments.

PREPARATION OF ANTIGEN Fresh thymus was obtained from young calves. It was kept on ice, and with minimal delay dissected clean, cut into small pieces, and homogenized in phosphate buffered saline (pH 7.3), 20\% wet weight/volume, using a glass homogenizer. After centrifugation (15-25 min. at 6,000 rpm), the supernatant was emulsified with an equal volume of FCA (Common- wealth Serum Laboratories, or Difco Ltd.). Emulsification was checked by the 'water drop' method, and the antigen was then used for immunization immediately. Antigens from rat and guinea-pig thymus or muscle were prepared using similar techniques. In some cases, the homogenate was initially lyophilized (Goldstein and Hofmann, 1968) to quantitate better antigen dosage. In the case of two groups of guinea-pigs (see Results), antigen preparation and injection were performed by Dr. Senga Whittingham, in the manner of Goldstein and Whittingham (1966).

IMMUNIZATION All animals, with the exception of those in two groups received $0.1 \mathrm{ml}$. of emulsion injected into the fore- or hind-foot pads. One group of animals received $\mathbf{0 . 2} \mathrm{ml}$. into each hind limb, and a second group received a 'booster' immunization one week after the primary injection of $0.1 \mathrm{ml}$. into the foot pad.

ELECTROMYOGRAPHY The animals were anaesthetized with pentobarbitone sodium $(35 \mathrm{mg} / \mathrm{kg})$. Electromyograms (EMGs) were recorded from plantar, flexor digitørum, and gastrocnemius muscles, through stainless steel needle electrodes. One needle was passed transversely through the full thickness of the muscle belly and the other was inserted more remotely. The limb was immobilized by clamps applied to the joints, which reduced but did not eliminate completely the movements caused by muscle contraction. The recording electrodes were connected to the input of a Tektronix 122 preamplifier, and the action potentials were displayed on the screen of a Tektronix 502A oscilloscope. The median or sciatic nerve was stimulated supramaximally through pairs of needle electrodes, inserted into the axillary fold or sciatic notch respectively. The stimulus was a square wave pulse of $0.2 \mathrm{msec}$ duration derived from a Devices Mark II stimulator. A number of animals were partially curarized while recordings were made; a progressive decline in EMG amplitude with repetitive stimulation was always obtained, indicating that the muscle action potentials were evoked by a neuromuscular mechanism rather than by direct muscle stimulation. 
Photographic records were made on $35 \mathrm{~mm}$ film, and measured after enlargement. Neuromuscular transmission was assessed by measuring the amplitude of the first and the tenth of the EMGs evoked by repetitive nerve stimulation at $50 / \mathrm{sec}$, although other stimulus frequencies were sometimes used in addition. The amplitude of the tenth response was expressed as a percentage of the amplitude of the first response. In two groups of animals, the effect on neuromuscular transmission of neostigmine methylsulphate, $0.2 \mathrm{mg} / \mathrm{kg}$, injected through a catheter in the internal jugular vein, was assessed.

MUSCLE TENSION In one group of animals, an assessment was made of gastrocnemius muscle tension development during repetitive sciatic nerve stimulation using the apparatus described by Brennan, Jones, and McLeod (1971).

DELAYED HYPERSENSITIVITY In guinea-pigs, delayed hypersensitivity was diagnosed by the presence of a slowly developing, indurated, necrotizing skin reaction after intradermal injection of $0.1 \mathrm{ml}$. of homogenate in buffered saline into the immunized animals and controls. In rats, necrotic lesions do not readily result from delayed hypersensitivity reactions (Humphrey and White, 1970). Progress measurements of skin thickness at the injection sites were made at four, 24 , and 48 hours after separate intradermal injections of antigen, and normal saline control solution, into both immunized and control animals (Nelson and Boyden, 1964).

SEROLOGICAL TESTING Sera from immunized and control guinea-pigs were diluted 1 in 15 with normal saline and tested for the presence of antibodies to thymic lymphocytes by means of the indirect fluorescent antibody test of Weller and Coons (1954). $4 \mu$ frozen sections of calf thymus were cut, and antigen-antibody reaction was demonstrated using rabbit anti-guinea-pig globulin labelled with fluorescein isothiocyanate. Rat sera were similarly tested, using serum dilutions of $1: 10$ and $1: 15$, $4 \mu$ frozen sections of rat thymus, and rabtit fluoresceinlabelled anti-rat globulin.

ThymiC HISTOLOGY After the final EMG studies, the thymus was removed from the rat or guinea-pig, and fixed in $10 \%$ formol saline. After dehydration, the tissue was embedded in paraffin, and $6 \mu$ sections were stained with haematoxylin and eosin.

Slides from immunized and control animals were studied together without prior knowledge of the group from which they were derived. Thymitis was considered to be present if there were focal accumulations of medium and small lymphocytes surrounding Hassall's corpuscles in the central medulla (Goldstein and Whittingham, 1967).

\section{RESULTS}

GUINEA-PIGS

NEUROMUSCULAR TRANSMISSION The results of the experiments are summarized in Table 1.
Initially, 16 guinea-pigs were immunized with calf thymus prepared and injected as described by $\frac{\bar{O}}{\circ}$ Goldstein and Whittingham (1966) (Table 1, group1). Neuromuscular transmission was assessed at one two, and three weeks after immunization by record- $D$ ing the EMG from the plantar muscles of the hindlimb, evoked by sciatic nerve stimulation at a rate $\frac{\rho}{\mathrm{S}}$. of $50 / \mathrm{sec}$. For each animal the amplitude of the $\frac{\overrightarrow{0}}{3}$ tenth muscle action potential evoked at a rate of $50 / \mathrm{sec}$ was recorded as a percentage of the amplitude $\overrightarrow{\bar{F}}$ of the first response of the series. Before immunization, the mean for the group was $85 \cdot 2 \%$ (SE 6.6). Two weeks after immunization, the value was $90 \cdot 1 \% \overline{\bar{c}}$. (SE 7.1), and at three weeks $92 \cdot 6 \%$ (SE 3.3). There $\widetilde{\Phi}$ was thus no development of the decremental response in muscle action potential amplitude on repetitive ${ }^{\infty}$ stimulation which characterizes myasthenia gravis. $\overrightarrow{0}$ Similar results were obtained when stimulus fre- $\overrightarrow{-}$ quencies of $1,3,10,20$, and $30 / \mathrm{sec}$ were used in assessing neuromuscular transmission.

In another group of animals similarly immunized with calf thymus (group II), no significant change was $\omega$ observed in the amplitude of the muscle action $+\vec{p}$ potentials or in the tension developed in the gastros nemius muscle.

In a third group of animals (group III), a secoffo을 injection of thymus antigen was administered oReweek after the first immunization and in this growp of animals action potentials were recorded from theo flexor digitorum muscle. Again no abnormality found electromyographically, nor was it in another group of animals (group IV) which had been inmunized with guinea-pig thymus instead of calf thymus.

In two groups of animals (groups $\mathrm{V}$ and VI) antigen preparation and injection were kindly per-O formed by Dr. Senga Whittingham using the methodo described by Goldstein and Whittingham (1966). No electrophysiological evidence of myasthenia was $\overrightarrow{0}$ obtained in thes $\epsilon$ animals, and no significant change 3 in the response to repetitive stimulation occurred after injection of neostigmine (Table 2).

DELAYED HYPERSENSITIVITY Five guinea-pigs from? each of groups I, III, and IV were tested two weekso after immunization for delayed hypersensitivity. (see Methods). All developed indurated necrotico lesions at the test sites. None of the control animals developed such lesions.

SEROLOGY Sera taken two weeks after immuniza: tion from 15 guinea-pigs selected variously from groups II, III, and VI (Table 1), and sera from aO group of 10 control guinea-pigs were studied at an dilution of 1 in 10 for the presence of antibodies ton calf thymic lymphocytes. Immunofluorescence tech 
TABLE 1

EFFECT OF REPETITIVE NERVE STIMULATION (50/SEC) ON AMPLITUDE OF MUSCLE ACTION IN ANIMALS IMMUNIZED WITH THYMUS OF MUSCLE IN FCA

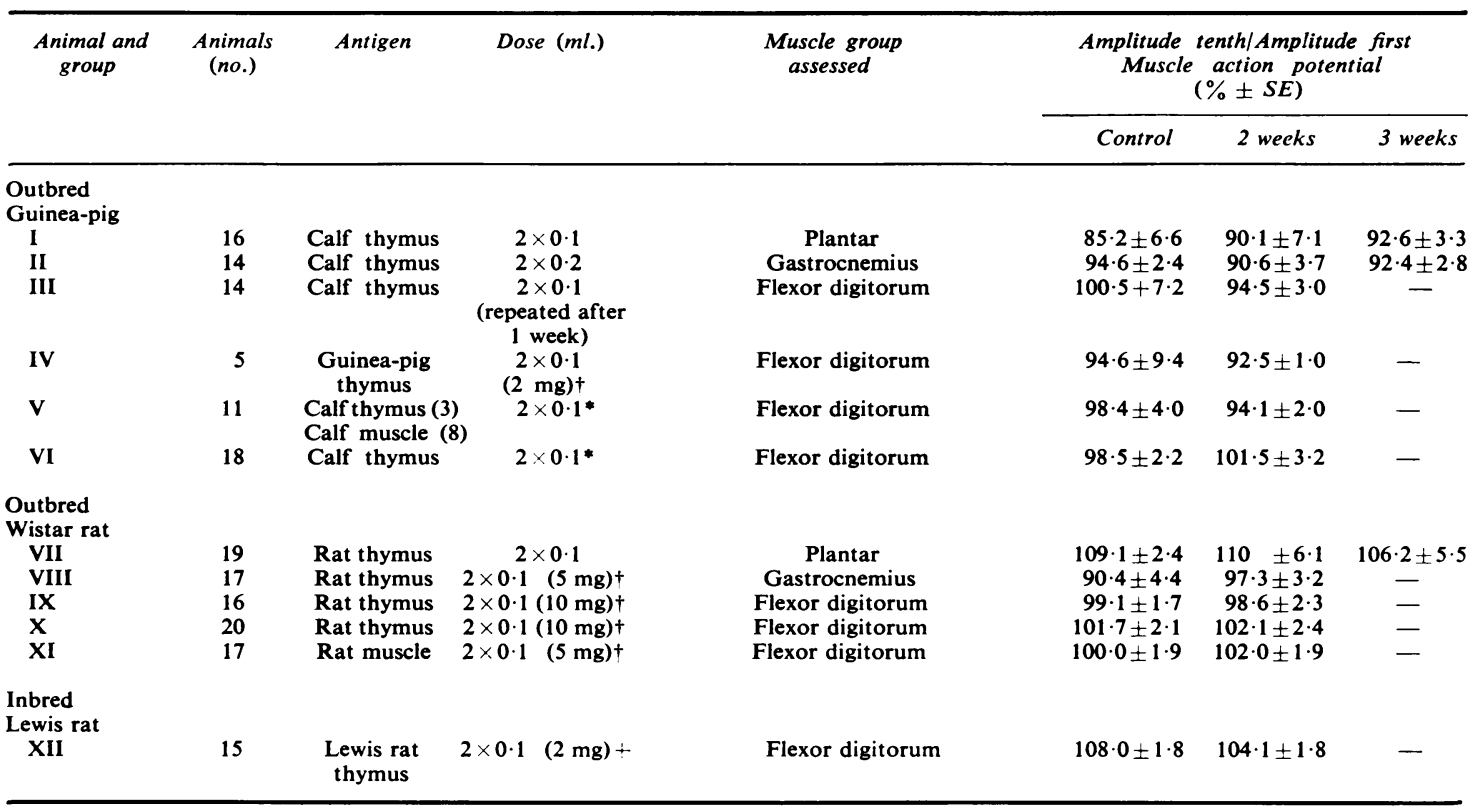

*Antigen preparation and injection kindly performed by Dr. Senga Whittingham. †Weight of lyophilized antigen used.

TABLE 2

EFFECT OF NEOSTIGMINE METHYLSULPHATE

(0.2 MG/KG) ON MUSCLE ACTION POTENTIALS EVOKED BY SINGLE STIMULI AND REPETITIVE STIMULATION (50/SEC) IN IMMUNIZED ANIMALS

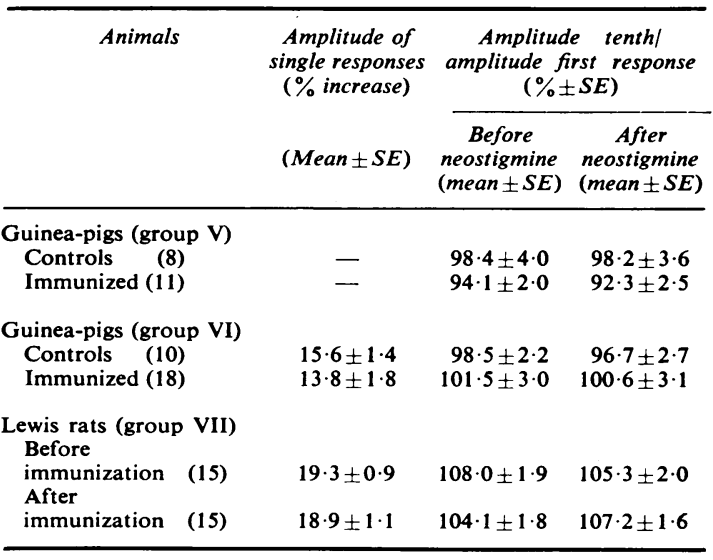

niques were used (see Methods). Slides were studied for fluorescence without the knowledge of the group of animals from which they were obtained. Thirteen of the 15 sera from immunized guinea-pigs were judged to contain anti-thymus antibodies. Two of the 10 control sera were also interpreted as containing anti-thymus antibodies.

THYMUS HISTOLOGY Sections from the thymuses of the $\mathbf{2 0}$ calf-thymus immunized guinea-pigs (groups $\mathrm{V}$ and VI, Table 1), from 10 control guinea-pigs immunized with FCA and saline only, and from 17 non-immunized guinea-pigs were studied (see Methods).

Histological features associated with thymitis were seen in 14 of the 27 control animals, and in 17 of the 20 immunized animals (Fig.). The difference in the incidence of thymitis in the two groups was statistically significant $(P<0.05$, chi square test $)$.

\section{RATS}

NEUROMUSCULAR TRANSMISSION The results are summarized in Table 1.

Outbred rats of the Wistar strain were immunized with rat thymus antigen and the muscle action potentials were recorded from plantar muscles. 


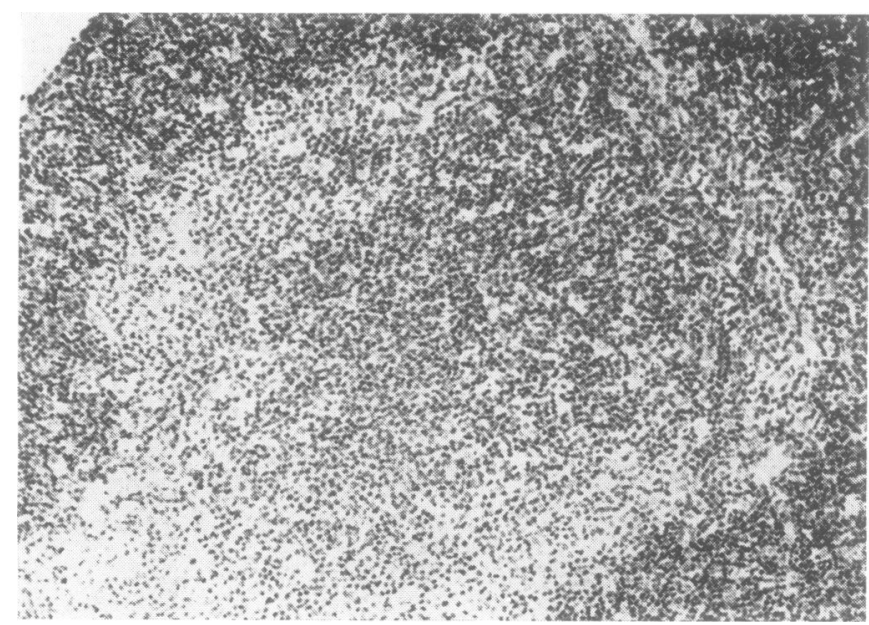

FIG. Guinea-pig thymus. Two weeks after immunization with calf thymus in FCA. There is an accumulation of lymphocytes in the thymic medulla, particularly around Hassall's corpuscles. $\times 110$. gastrocnemius muscles, and flexor digitorum muscles two weeks after immunization, and in the case of group VII also at three weeks after immunization. Another group of Wistar rats, group XI, was immunized with antigen obtained from rat muscle. In none of the groups of animals was electrophysiological evidence of myasthenia demonstrated (Table 1). Finally, a group of inbred Lewis rats (group XII) was immunized with antigen obtained from Lewis rat thymus. Again, no electrophysiological abnormalities were demonstrated (Table 1), and no alteration in the response followed injection of neostigmine (Table 2).

DELAYED HYPERSENSITIVITY Five rats from each of groups $\mathrm{X}$ and $\mathrm{XI}$ were also tested for delayed hypersensitivity two weeks after immunization. None developed necrotic lesions at the test sites. Measurements at 24 and 48 hours after the test innoculations showed a greater mean increase in skin fold thickness in immunized than in control animals, but the difference was not significant.

SEROLOGY Sera from five rats of group IX, and five rats of group XII, and from 10 control (nonimmunized) rats were studied at dilutions of $1: 10$ and 1:15 for the presence of antibodies to rat thymic lymphocytes (see Methods). No differences could be detected between sera from control and immunized animals.

THYMUS HISTOLOGY The thymuses of eight out of 20 control rats showed features which could be interpreted as thymitis, and the thymuses of 18 of 24 immunized animals in groups VII and XII showed similar changes (Table 3). There was a significant difference in the incidence of thymitis in the two groups $(P<0.05$, chi square test $)$.
TABLE 3

NUMBERS OF ANIMALS WITH PATHOLOGICAL FEATURES CONSISTENT WITH THYMITIS

\begin{tabular}{|c|c|c|c|}
\hline Animals & Total number & $\begin{array}{c}\text { Number with } \\
\text { thymitis }\end{array}$ & $\begin{array}{l}\text { Significance } \\
\text { of difference }\end{array}$ \\
\hline $\begin{array}{l}\text { Guinea-pigs } \\
\text { Controls } \\
\text { Immunized }\end{array}$ & $\begin{array}{l}27 \\
20\end{array}$ & $\begin{array}{l}14 \\
17\end{array}$ & $P<0.05$ \\
\hline $\begin{array}{l}\text { Rats } \\
\text { Controls } \\
\text { Immunized }\end{array}$ & $\begin{array}{l}20 \\
24\end{array}$ & $\begin{array}{r}8 \\
18\end{array}$ & $P<0.05$ \\
\hline
\end{tabular}

DISCUSSION

The foregoing results represent a consistent failure $\bar{\partial}$ to detect a myasthenic defect in neuromuscular $\frac{\not}{\varnothing}$ transmission in groups of animals immunized by the $\varrho$ methods described by Goldstein and his co-workers. $\overrightarrow{\overrightarrow{0}}$

The possibility that our failure to detect an abnor- 3 mality may be partly due to differences in the techniques used in assessing neuromuscular transmission must be considered. The needle electrodes used in the present study are more suitable for recording myasthenic responses than the coaxial needle electrodes employed by Goldstein and Whittingham (1966), since they are less likely to give rise to movement $\delta$ artefacts (Vetters et al., 1969). Kaufman et al. (1969), $₹$ who used an electrode system similar to that em- o ployed by Goldstein and Whittingham (1966) commented that artefactual recordings due to displacement of intramuscular electrodes were frequently encountered. However, Kalden and Irvine (1969) $\Omega$ were satisfied that their recordings of myasthenic $N$ responses in immunized rats were not artefactually N produced. In the present study, isometric muscle 
tension was recorded directly in one group of immunized animals, but again no evidence of myasthenia was found using this technique. The methods of assessing neuromuscular transmission by comparing the amplitude of the tenth muscle action potential with the first of a series evoked by repetitive stimulation at a rate of 50 per second, and by assessing the effect of neostigmine are similar to those employed by Goldstein and Whittingham (1966) and Kalden et al. (1969). The possibility that the recorded muscle activity resulted from direct stimulation of muscle was excluded by observing the effects of partial curarisation. The muscles studied in our experiments included those studied by these authors, and other muscle groups in addition. Muscle function was tested in groups I, II, and VII at intervals of three weeks after immunization in addition to the usual assessment at two weeks.

Another possible explanation of our failure to produce myasthenia is that our immunization procedures did not produce autoimmune thymitis in the particular experimental animals used. However, heterologous, homologous, and isologous antigens, and FCA from two separate sources have been used. Antigen has been prepared, and animals immunized on many separate occasions in the manner detailed by Goldstein and co-workers. It appears unlikely that the results represent a technical failure to reproduce the reported methods of antigen preparation and injection; the failure of the guinea-pigs immunized by Dr. S. Whittingham to develop myasthenia strongly supports this conclusion. Furthermore, the hypersensitivity and serological studies indicate that, at least with guinea-pigs, appropriate immunological responses were initiated. The failure to detect anti-thymus antibodies in the sera of homologously immunized rats does not contradict this, being consistent with the failure of Goldstein et al. (1969) to detect such antibodies in homologously immunized guinea-pigs.

Our investigations of thymic histology support reports that immunization with thymus in FCA increases the incidence of a histological picture characterized by focal lymphocytic accumulations in the thymic medulla. However, we agree with Webb (1969) and Vetters et al. (1969) that a similar picture is also frequently a normal finding, since appearances consistent with thymitis were present in approximately one half of our control animals.

An explanation of our failure to confirm the findings of Goldstein and his co-workers may be that the development of myasthenia, and, perhaps, autimmune thymitis of sufficient intensity, is a highly specific phenomenon for certain strains of experimental animals. Animals used in the present investigations may have had low susceptibility to development of the immunological lesions. However, the absence of myasthenia in guinea-pigs receiving as much as $10 \mathrm{mg}$ of lyophilized antigen on repeated immunization and our inability to produce the lesion in inbred Lewis rats probably genetically close to those used by Goldstein and Hofmann (1966) seem to be evidence against such an explanation.

Our findings fail to confirm the observation of Goldstein and Whittingham (1966), and Kalden et al. (1969) but are in agreement with those of Vetters et al. (1969) and Kaufman et al. (1969). Further investigation will need to be undertaken if immunized rats and guinea-pigs are to be regarded and used as reliable experimental models of the human disease of myasthenia gravis.

The work was supported by a grant from The National Health and Medical Research Council of Australia. Miss Julia Baverstock gave valuable technical assistance.

\section{REFERENCES}

Brennan, J. L., Jones, S. F., and McLeod, J. G. (1971). Effect of germine acetates on neuromuscular transmission. $J$. neurol. Sci. (In press).

Goldstein, G., and Whittingham, S. (1966). Experimental autoimmune thymitis. An animal model of human myasthenia gravis. Lancet, 2, 315-318.

Goldstein, G., and Whittingham, S. (1967). Histological and serological features of experimental autoimmune thymitis in guinea pigs. Clin. exp. Immunol., 2, 257-268.

Goldstein, G., and Hofmann, W. W. (1968). Electrophysiological changes similar to those of myasthenia gravis in rats with experimental autoimmune thymitis. J. Neurol. Neurosurg. Psychiat., 31, 453-459.

Goldstein, G., Strauss, A. J. L., and Pickeral, S. (1969). Antigens in thymus and muscle effective in inducing experimental autoimmune thymitis and the release of thymin. Clin. exp. Immunol., 4, 3-16.

Humphrey, J. H., and White, R. G. (1970). Immunology for Students of Medicine. 3rd edn. Blackwell: Oxford.

Kalden, J. R., and Irvine, W. J. (1969). Experimental myasthenia gravis. Lancet, 2, 638-9.

Kalden, J. R., Williamson, W. G., Johnston, R. J., and Irvine, W. J. (1969). Studies on experimental autoimmune thymitis in guinea-pigs. Clin. exp. Immunol., 5, 319-340.

Kaufman, B. M., Rushworth, G., and Wright, R. (1969). Experimental studies related to autoimmunity in myasthenia gravis. J. Neurol. Neurosurg. Psychiat., 32, 281-289.

Nelson, D. S., and Boyden, S. V. (1964). The cutaneous reactivity of guinea-pigs to pure protein antigens. Int Arch. Allergy, 25, 279-303.

Vetters, J. M., Simpson, J. A., and Folkarde, A. (1969). Experimental myasthenia gravis. Lancet, 2, 28-31.

Webb, J. N. (1969). Experimental myasthenia gravis. Lancet, 2, 803.

Weller, T. H., and Coons, A. H. (1954). Fluorescent antibody studies with agents of varicella and herpes zoster propagated in vitro. Proc. Soc. exp. Biol. (N.Y.), 86, 789-794. 